\title{
The effects of rehearsal on temporal order coding
}

\author{
MOSHE NAVEH-BENJAMIN \\ Ben-Gurion University of the Negev, Beer-Sheva, Israel
}

\begin{abstract}
It has been claimed that temporal order information is encoded automatically. This claim has not been uniformly supported by experimentation that has included tests of several criteria suggested for identifying automatic processes. The present experiment extends these tests to a case in which temporal order judgments were obtained in a rehearsal task with massed presentation. In this task, temporal order coding was sensitive to strategy manipulations. In fact, in one condition there was no reliable evidence of any temporal order coding. These results contradict the claim that temporal order coding is automatic.
\end{abstract}

In recent years, there has been a growing interest in the study of automatic processes. One frequently cited example of such processes is the encoding of temporal order information. Hasher and Zacks $(1979,1984)$ have argued that people automatically code the order in which events occur. To test whether temporal order information is encoded automatically, several researchers have examined a number of criteria that must be satisfied by a process if it is to be considered automatic. The results of these studies are mixed. Some studies have shown that temporal order information is stored under incidental learning conditions (Hintzman \& Block, 1971; Proctor \& Ambler, 1975). Other studies have shown that temporal order encoding is not enhanced by intention to code temporal information (e.g., Toglia \& Kimble, 1976; Zimmerman \& Underwood, 1968), nor is it influenced by competing task demands (Zimmerman \& Underwood, 1968) or by age (Brown, 1973; Perlmutter, Metzger, Nezworski, \& Miller, 1981). Other studies, however, have shown temporal order information to be affected by intention (Jackson \& Michon, cited in Michon \& Jackson, 1984; NavehBenjamin, 1990), by competing task demands (NavehBenjamin, 1990), and by age (McCormack, 1982; NavehBenjamin, 1990). These contradictory results seem to be the result of the operational definitions of some of the criteria in these studies. The varied definitions make it difficult to reach definite conclusions about the automaticity of temporal order coding (see Naveh-Benjamin, 1990 , for elaboration).

The major assumption underlying all of the criteria tested is that automatic processes should function at a constant level under all circumstances. Thus, if temporal order coding is an automatic process, we would expect that manipulating the coding strategy would not affect the fidelity of temporal order judgments.

I would like to thank Sigalit Rozenzweig and Rinat Shemes for their help in data collection and analysis. Requests for reprints may be addressed to Moshe Naveh-Benjamin, Department of Behavioral Sciences, Ben-Gurion University of the Negev, P.O. Box 653, Beer-Sheva 84120, Israel.
The purpose of the present experiment was to test whether variation in encoding strategy has an effect on temporal order judgments. To render this a powerful test, two encoding strategies were intentionally confounded. The first strategy was the level of processing used by the subjects while encoding the information: In one condition, the subjects created a mental image of each word presented; in the second condition, the subjects simply repeated the words aloud. The second encoding strategy was the intention to learn the information: In the condition in which the subjects used mental imagery, they were also instructed to remember the words for a temporal order memory test; in the condition in which they only repeated the words, they were not led to expect a memory test. If automatic processes function at a constant level under all circumstances, as Hasher and Zacks (1979) have claimed, we should expect no difference between the temporal order judgments of the two groups.

This variation in encoding strategy was implemented in a task that varied the number of times that subjects rehearsed the words whose temporal order was later tested. The basic task was a modification of the classic Brown-Peterson short-term memory task (Glenberg, Smith, \& Green, 1977). In each trial, subjects were given a set of three two-digit numbers to hold in memory. During the retention interval, they were presented with a word. One group was told to create an image of the word in preparation for an upcoming temporal order memory test for the word and to elaborate this image as they rehearsed the word aloud. The other group was told simply to repeat the word aloud and was told nothing about a memory test. For both groups, the number of overt rehearsals was one, three, or seven. After a series of such trials, we queried subjects from both groups about the order in which the words appeared-their temporal order. If temporal order is automatically coded, there should have been no difference in performance of the groups on the temporal order test.

\section{METHOD}

\footnotetext{
Subjects

Thirty students at Ben-Gurion University served as paid volunteers in a session of approximately 40-min duration.
} 


\section{Design}

The experiment included two major independent variables. The first, which varied within subjects, was the number of rehearsals required on each trial (one, three, or seven). The second, which varied between subjects, was the instruction under which rehearsal occurred. In one condition, the subjects were told simply to repeat the words; in the other, they were told to create an image of each word and to elaborate these images in preparation for a later temporal order memory test. The dependent variables were the proportion correct on the numbers test and the percentage correct on a pair-judgments temporal order test for the words.

\section{Apparatus}

The numbers and the words were presented using a tape recorder. The subjects sat in separate booths with headphones and a small microphone that was placed in front of each of them. The booths were arranged so the subjects did not interfere with each other's rehearsals.

\section{Stimuli}

Two types of stimuli were used: numbers and words.

Numbers. From the subject's point of view, the main experimental task was to memorize and recall sets of three two-digit numbers that were selected randomly for each subject with the following constraints: all stimuli were drawn from the numbers $12-98$, inclusive; no numbers with two identical digits were used (e.g., 66); no two numbers in a set were chosen from the same decade; and no two numbers in a set were integer multiples of one another.

Words. Throughout each interval during which the numbers were to be retained, the subjects were required to recite aloud (rehearse) a word. The words chosen for rehearsal were one-, two-, or three-syllable Hebrew words four to six letters long and of greater frequency than 20 occurrences per million (Kucera \& Frances, 1967). The words were presented in random order.

\section{Temporal Order Test}

The test of temporal order was administered after all trials were completed. The 30 rehearsed words were paired in the following way. One third of the pairs included words that appeared in trials that were separated by one to four other trials (short distance). One third of the pairs included words that appeared in trials that were separated by five to nine other trials (medium distance). The last third of the pairs included words that appeared in trials separated by 10 trials or more (long distance). The order between pairs as well as within pairs was randomized. For each pair of words, the subjects were asked to circle the word that appeared later in the sequence of the words in the learning stage. To avoid confounding order with number of rehearsals, words in each pair in the test were matched for the number of times they were rehearsed in the learning phase.

\section{Trial Structure}

There were 40 trials in the experiment. Of these, the first four were considered practice and were not analyzed. The next three trials after the practice trials and the last three trials of the experiment were also excluded from analysis to minimize the effects of primacy and recency in the later temporal order test. This left 30 test trials, 10 for each value of rehearsal interval. The subjects rehearsed items one, three, or seven times on different trials. For each trial, the following sequence of events occurred.

First, a warning tone was presented for $0.5 \mathrm{sec}$. Following this tone, three two-digit numbers were presented at a rate of one number per second. Half a second after the last number was presented, a word was presented. Following presentation of the word, the subjects heard a set of tones. The tones occurred at a rate of one every $1.2 \mathrm{sec}$. One, three, or seven tones were presented. Each length of tone sequence occurred 10 times, and the various lengths were distributed randomly in a session to prevent the subjects from predicting the length of the rehearsal interval. Following the tones, two brief tones were presented; this prompted the subjects to write down their recall of the three numbers. They had $10 \mathrm{sec}$ to do so before the next trial was initiated.
Procedure

The subjects were run in groups of 2 to 4 subjects each. The subjects in the incidental-maintenance rehearsal condition were told that they were in an experiment on short-term memory for digits and that they would have to repeat words aloud as a distractor activity between presentation and recall of the digits. They were not told about any test of the words. The subjects in the intentional-elaborative rehearsal condition were told about the upcoming temporal order memory test for the words and its nature, and they were urged to rehearse the words elaboratively (using a mental image for each word) to help them remember the words' temporal order. The subjects were cautioned, however, to keep their recall of the numbers at a reasonable level. Both groups were told to rehearse each word into a microphone at a pace set by the tones. Following the experimental trials, the subjects were engaged in $2 \mathrm{~min}$ of interpolated unrelated activity, after which the temporal order test was administered. The subjects had as much time as they needed to complete the test.

\section{RESULTS}

\section{Number Recall}

Table 1 presents the percentage of numbers recalled correctly as a function of rehearsal condition and number of rehearsals. A strict criterion was used in which a response was scored as correct only if all three two-digit numbers were recalled in their correct order of presentation. The analysis showed that recall performance was higher for the incidental-maintenance rehearsal condition, although this advantage did not reach statistical significance $[F(1,28)=0.34$, n.s.]. This advantage is not surprising, because the subjects in the intentionalelaborative rehearsal condition were presumably devoting more effort to learning the words than were the subjects in the incidental-maintenance rehearsal condition. Another indication for differences in resource-allocation policies in the different conditions was obtained in a postexperiment questionnaire. The subjects in the incidental-maintenance condition indicated that they spent $95 \%$ of their resources on memorizing the numbers, whereas those in the intentional-elaborative condition spent only $65 \%$ of their resources on this task. Recall performance also dropped significantly with increasing number of rehearsals $[F(2,56)=4.69, p<.02]$. This effect has been found in some other rehearsal studies (e.g., Naveh-Benjamin \& Jonides, 1984a, 1984b). The interaction of rehearsal condition and number of rehearsals did not reach statistical significance $[F(2,56)=0.81$, n.s. $]$.

\section{Temporal Order Judgments}

For each subject, the percentage of correct choices of whether an item appeared later in the learning stage was

Table 1

Effects of Rehearsal Condition and Number of Rehearsals on Percentage of Numbers Recalled Correctly

\begin{tabular}{|c|c|c|c|c|c|c|}
\hline \multirow[b]{3}{*}{ Rehearsal Condition } & \multicolumn{6}{|c|}{ Number of Rehearsals } \\
\hline & \multicolumn{2}{|c|}{1} & \multicolumn{2}{|c|}{3} & \multicolumn{2}{|c|}{7} \\
\hline & $M$ & $S D$ & $M$ & $S D$ & $M$ & $S D$ \\
\hline Incidental-Maintenance & 54.0 & 23.8 & 54.0 & 21.9 & 42.0 & $\begin{array}{l}24.8 \\
23.9\end{array}$ \\
\hline Intentional-Elaborative & 50.0 & 21.0 & 45.3 & 23.5 & 42.0 & 23.9 \\
\hline
\end{tabular}


calculated separately for each temporal distance. The means of these measures across each group of subjects are presented in Table 2. A two-way analysis of variance revealed a significant effect of rehearsal condition $[F(1,28)=7.00, p<.02]$ and a significant interaction of rehearsal condition with distance $[F(2,56)=3.71$, $p<.05]$. The effect of distance, which showed the expected better overall performance with larger distance, was not statistically significant $[F(2,56)=0.02$, n.s. $]$. This lack of significance was mostly due to the trends in the incidental-maintenance rehearsal condition, where performance was better in the short-distance pairs.

Overall, these results indicate that the subjects in the intentional-elaborative rehearsal condition could better discriminate among the temporal order of the words. Furthermore, the results indicate that performance in the incidental-maintenance rehearsal condition $(46.7 \%)$ was not at a better than chance level, which implies that the subjects in this condition could not reliably discriminate among the temporal order of any of the words.

\section{DISCUSSION}

The results of the above experiment are quite clear. The subjects in the intentional-elaborative rehearsal condition showed superior temporal order judgments over the subjects in the incidental-maintenance rehearsal condition. Such results contradict one of the major criteria suggested by Hasher and Zacks (1979) for automatic processes, and they extend the conditions under which it has been shown that strategy manipulations, such as intention to learn or levels of processing, affect temporal order judgments (Naveh-Benjamin, 1990).

What causes performance differences between the conditions? There are two obvious variables: intention to learn and level of processing. Some studies have found no effect of intention (e.g., Toglia \& Kimble, 1976; Zimmerman \& Underwood, 1968); however, those studies compared intentional instructions stressing temporal order registration with incidental learning instructions that stressed item memory. The apparent lack of difference between the two conditions in those studies could be due to the fact that inappropriate incidental learning instructions were used. A true incidental learning condition in which subjects do not expect any memory test on the information presented should be used, so that learning could be considered truly incidental. This type of manipulation was shown in the experiment reported here, as well as elsewhere (Naveh-Benjamin, 1990), to have sizable effects on memory for temporal order information.

The other variable that could lead to performance differences between the two conditions is the level of processing used by the subject. Jackson, Michon, Boonstra, De Jonge, and De Velde Harsenhorst (1986) examined the effects of different levels of processing of words on memory for temporal order information. Their results showed that relative temporal order information (as reflected in pair judgments) was more accurate when subjects processed semantic features of the information than when they processed word-structure information. That study, however, used a shallow-level physical task (cf. Craik \& Lockhart, 1972) in which subjects had to judge whether a given letter appeared in each word. Such

Table 2

Effects of Temporal Distance and Rehearsal Condition on Percentage of Correct Temporal Order Judgments

\begin{tabular}{|c|c|c|c|c|c|c|}
\hline \multirow[b]{3}{*}{ Rehearsal Condition } & \multicolumn{6}{|c|}{ Temporal Distance } \\
\hline & \multicolumn{2}{|c|}{ Short } & \multicolumn{2}{|c|}{ Medium } & \multicolumn{2}{|c|}{ Long } \\
\hline & $M$ & $S D$ & $M$ & $S D$ & $M$ & $S D$ \\
\hline Incidental-Maintenance & 53.3 & 24.0 & 40.0 & 21.6 & 46.7 & 24.7 \\
\hline Intentional-Elaborative & 51.7 & 32.0 & 68.8 & 16.5 & 67.4 & 25.3 \\
\hline
\end{tabular}

a task does not require any processing of the words as whole units, so it is not surprising that word-order judgments were better under semantic processing instructions. In the current research (as well as in the one by Naveh-Benjamin, 1990), there was a clear effect of levels of processing on memory for temporal order when instructions for both groups required processing the words as whole units.

Even more striking are the results for the incidental-maintenance rehearsal condition. The performance of the subjects in this condition in the temporal order task did not reliably differ from chance, so the hypothesis that these subjects did not encode any information that enabled them to discriminate the temporal order of items cannot be rejected. Such results clearly contradict a major assumption about automatic processes-that they function at an optimal level under all circumstances. At the very least, this assumption seems to describe temporal order coding inappropriately. As discussed elsewhere (e.g., NavehBenjamin, 1987, 1988; Naveh-Benjamin \& Jonides, 1986), it may be too stringent an assumption for automaticity assessment in general.

Note that the context in which the experiment reported here was done-a rehearsal paradigm-should have enhanced temporal order judgments. This is so because words were repeated successively more than once and there was a filled interval of about $20 \mathrm{sec}$ between the appearances of each of the words. This should have increased the probability of marking their temporal order. Nevertheless, it did not help the subjects in the incidental-maintenance rehearsal condition encode any information about the temporal order of the words.

Also note that the test used here-pair judgment-relies less on retrieval processes than does an absolute test of temporal order in which subjects are requested to arrange words according to their temporal order. This test, however, did not help the subjects in the incidentalmaintenance rehearsal condition in their temporal order judgments.

In sum, the results of the experiment reported here are clear: they do not support the claim for automaticity in encoding temporal order information, and they fit the results of other studies (on frequency of occurrence and spatial location, for instance) that also contradict the claim for automaticity. For example, studies by Fisk and Schneider (1984), Greene (1984, 1986), Jonides and Naveh-Benjamin (1987), and Naveh-Benjamin and Jonides $(1985,1986)$ have shown that frequency coding is influenced by intention, by competing task load, and by strategy manipulations. In addition, Naveh-Benjamin $(1987,1988)$ has shown that spatial location coding is influenced by intention, subjects' age, competing task loads, practice, strategy manipulation, and individual differences. These results do not support claims for automaticity of frequency, spatial, or temporal order information. As discussed elsewhere (e.g., Naveh-Benjamin, 1987, 1988), these results challenge the automaticity of these aspects of information processing as suggested by Hasher and Zacks (1979), as well as the criteria for automaticity and their underlying assumptions.

\section{REFERENCES}

Brown, A. L. (1973). Judgments of recency for long sequences of pictures: The absence of a developmental trend. Journal of Experimental Child Psychology, 15, 473-480.

Craik, F. I. M., \& LockHART, R. S. (1972). Levels of processing: A framework for memory research. Journal of Verbal Learning \& Verbal Behavior, 11, 671-684.

FisK, A. D., \& SCHNEIDER, W. (1984). Memory as a function of attention, level of processing, and automatization. Journal of Experimental Psychology: Learning, Memory, \& Cognition, 10, 181-197.

Glenberg, A., Smith, S. M., \& Green, C. (1977). Type I rehearsal: Maintenance and more. Journal of Verbal Learning \& Verbal Behavior, 16, 339-352.

GREENE, R. L. (1984). Incidental learning of event frequency. Memory \& Cognition, 12, 90-95.

GREENE, R. L. (1986). Effects of intentionality and strategy on memory for frequency. Journal of Experimental Psychology: Learning, Memory, \& Cognition, 12, 489-495.

HASHER, L., \& ZACKs, R. T. (1979). Automatic and effortful processes in memory. Journal of Experimental Psychology: General, 108, 356-388. 
HASHER, L., \& ZACKS, R. T. (1984). Automatic processing of fundamental information: The case of frequency of occurrence. American Psychologist, 39, 1372-1388.

Hintzman, D. L., \& Block, R. A. (1971). Repetition and memory: Evidence for a multiple-trace hypothesis. Journal of Experimental Psychology, 88, 297-306.

Jackson, J. L., Michon, J. A., Boonstra, H., De Jonge, D., \& De Velde HaRsenhorst, J. (1986). The effect of depth of processing on temporal judgment tasks. Acta Psychologica, 62, 199-210.

JoNIDES, J., \& NAVEH-BENJAMIN, M. (1987). Estimating frequency of occurrence. Journal of Experimental Psychology: Learning, Memory, \& Cognition, 13, 230-240.

KuČERA, H., \& Francis, W. (1967). Computational analysis of present day American English. Providence, RI: Brown University Press.

McCormack, P. D. (1982). Temporal coding and study-phase retrieval in young and elderly adults. Bulletin of the Psychonomic Society, 20, 242-244.

Michon, J. A., \& JACkson, J. L. (1984). Attentional effort and cognitive strategies in the processing of temporal information. Annals of the New York Academy of Sciences, 423, 298-321.

Naveh-Benjamin, M. (1987). Coding of spatial location information: An automatic process? Journal of Experimental Psychology: Learning, Memory, \& Cognition, 13, 595-605.

Naveh-Benjamin, M. (1988). Recognition memory of spatial location information: Another failure to support automaticity. Memory \& Cognition, 16, 437-445.

Naveh-Benjamin, M. (1990). Coding of temporal order information: An automatic process? Journal of Experimental Psychology: Learning, Memory, \& Cognition, 16, 117-126.
Naveh-Benjamin, M., \& Jonides, J. (1984a). Cognitive load and maintenance rehearsal. Journal of Verbal Learning \& Verbal Behavior, 23, 494-507.

NAVEh-Benjamin, M., \& Jonides, J. (1984b). Maintenance rehearsal: A two-component analysis. Journal of Experimental Psychology: Learning, Memory, \& Cognition, 10, 369-385.

NaVeh-Benjamin, M., Jonides, J. (1985). The effects of rehearsal on frequency coding. Bulletin of the Psychonomic Society, 23, 387-390.

NAVeh-Benjamin, M., Jonides, J. (1986). On the automaticity of frequency coding: Effects of competing task load, encoding strategy, and intention. Journal of Experimental Psychology: Learning, Memory, \& Cognition, 12, 378-386.

Perlmutter, M., Metzger, R., Nezworski, T., \& Miller, K. (1981). Spatial and temporal memory in 20 and 60 year olds. Journal of Gerontology, 36, 59-65.

Proctor, R. W., \& AMBler, B. A. (1975). Effects of rehearsal strategy on memory for spacing and frequency. Journal of Experimental Psychology: Human Learning \& Memory, 1, 640-647.

Toglia, M. P., \& Kimble, G. A. (1976). Recall and use of serial position information. Journal of Experimental Psychology: Human Learning \& Memory, 2, 431-445.

Zimmerman, J., \& Underwood, B. J. (1968). Ordinal position knowledge within and across lists as a function of instructions in freerecall learning. Journal of General Psychology, 79, 301-307.

(Manuscript received September 8, 1989.) 